\title{
Instalación y funcionamiento de un laboratorio de ensayos con la finalidad de un control propio de fábrica, en las factorías de hormigón
}

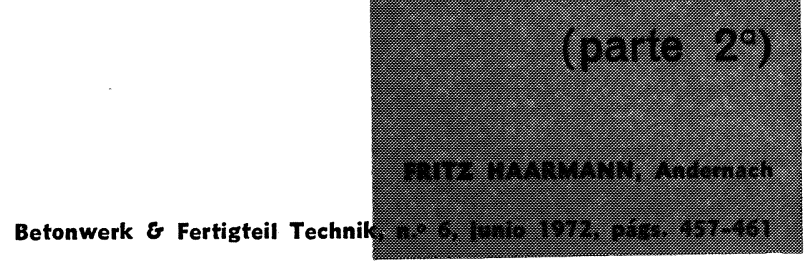

Continuación y conclusión.-La parte $1 .^{a}$ de este trabajo se publicó en el número 149 de esta Revista (enero-febrero-marzo 1973).

E) Más ejemplos de laboratorios de ensayos para campos de trabajo limitados o de mayor alcance

El laboratorio que aparece en la figura 1, Parte $1 .^{\mathrm{a}}$, no representa un prototipo único. No existe un laboratorio de ensayos uniforme. Cada uno de los laboratorios de ensayos, incluso el laboratorio $\mathrm{E}$, se regirá, en cuanto a su tamaño y equipo, por el tipo y volumen de programa de la fábrica o de las fabricas a las que hay que asistir y también, decisivamente, por la economía. A continuación presentamos otros ejemplos de laboratorios pequeños o grandes, resultado de contactos con especialistas, directores de laboratorio, asociaciones, observaciones y experiencia. Los símbolos correspondientes a cada una de las instalaciones son los mismos en todas para facilitar así una evaluación comparativa.

En la más pequeña factoría hay lugar para esto: Garaje prefabricado con puerta basculante. El fabricante montó una ventana a ambos lados laterales. El armario de aparatos de ensayo de la Asociación Alemana del Hormigón ha tenido en cuenta las estrechas dimensiones. Contiene el mínimo necesario de aparatos de comprobación para el ensayo de áridos y hormigón no fraguado. Su construcción, a modo de escritorio, lo convierte en el lugar de trabajo del ensayador. La caja de aire acondicionado, 24, sustituye al local con aire acondicionado. Toda la anchura de la pared posterior está ocupada por la mesa de trabajo. La máquína de ensayo, 13/14, se encuentra a la entrada donde resulta accesible a pesar del pequeño espacio. Instalación del local de ensayos al aire libre situada en el centro del terreno de la fábrica. Tendido subterráneo de las conexiones flexibles para la energía y el agua. Pozo de filtrado. Si posteriormente se traslada la oficina de ensayos a un local más amplio, estacionario, se podrá disponer nuevamente del garaje para su finalidad propiamente dicha. 


\section{En las figuras 2 a 9 los números representan:}

1 Conglomerantes o áridos.

2 Depósito de agua.

3 Mezcladora pequeña.

4 Mesa de sacudidas, cono y tamices según $O$. Graf.

5 Compactador según Walz.

6 Mesa vibradora.

7 Secadero.

8 Balanza.

9 Mesa de trabajo.

10 Repisas o armarios para utensilios.

11 Plano de descarga o desenmolde.

12 Escritorio.

13 Prensa para compresión.
14 Prensa para flexotracción.

15 Pupitre móvil.

16 Aparato para ensayo de tubos por presión hidráulica interna.

17 Contador del agua para el 16.

18 Aparato para el ensayo de tubos por presión externa.

19 Aparato para medir la permeabilidad al agua.

20 Mando de las prensas.

21 Tamizadora.

22 Puente-grúa.

23 Equipo de ensayos según la Asociación Alemana del Hormigón.

24 Armario o caja climatizada.

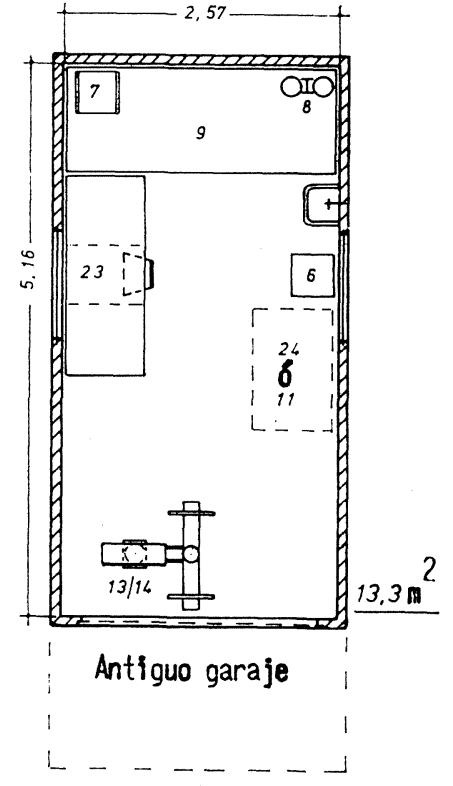

Fig. 2.-Laboratorio de ensayos muy pequeño para instalación dispuesta en voladizo. Tamaño del local: $13 \mathrm{~m}^{2}$.

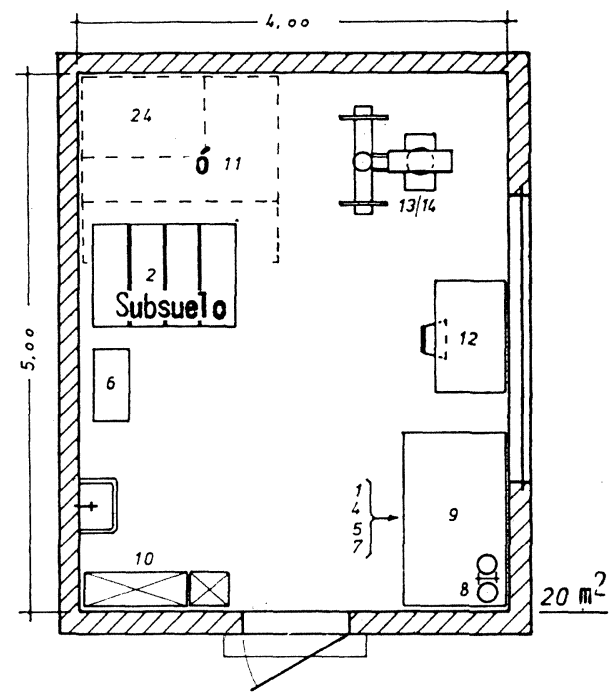

Fig. 3.-Laboratorio de ensayos anejo o interior. Tamaño del local: $20 \mathrm{~m}^{2}$.

Los aparatos de ensayo están bajo la mesa, 9, y en la estantería de la pared. El depósito de agua se halla bajo el suelo, 2. El almacenamiento al aire libre, bajo yute húmedo, 11; o instalación de un armario de aire acondicionado, 24. Mucha luz.

Instalación similar a la de la figura 1 (Parte $1 .{ }^{a}$ ), pero de suficiente magnitud: Local sin polvo para el equipo de ensayo de la presión dentro del agua, 16/17. Aparato de comprobación de la presión de cresta, 18, y evaluación, 12. Disposición funcional de los aparatos 
de ensayo de tubos. En primer lugar ensayos de la presión dentro del agua, y a continuación vuelco del tubo con la estibadora e introducirlo en la máquina de ensayo de la presión de cresta. Sólo un acceso de puerta.

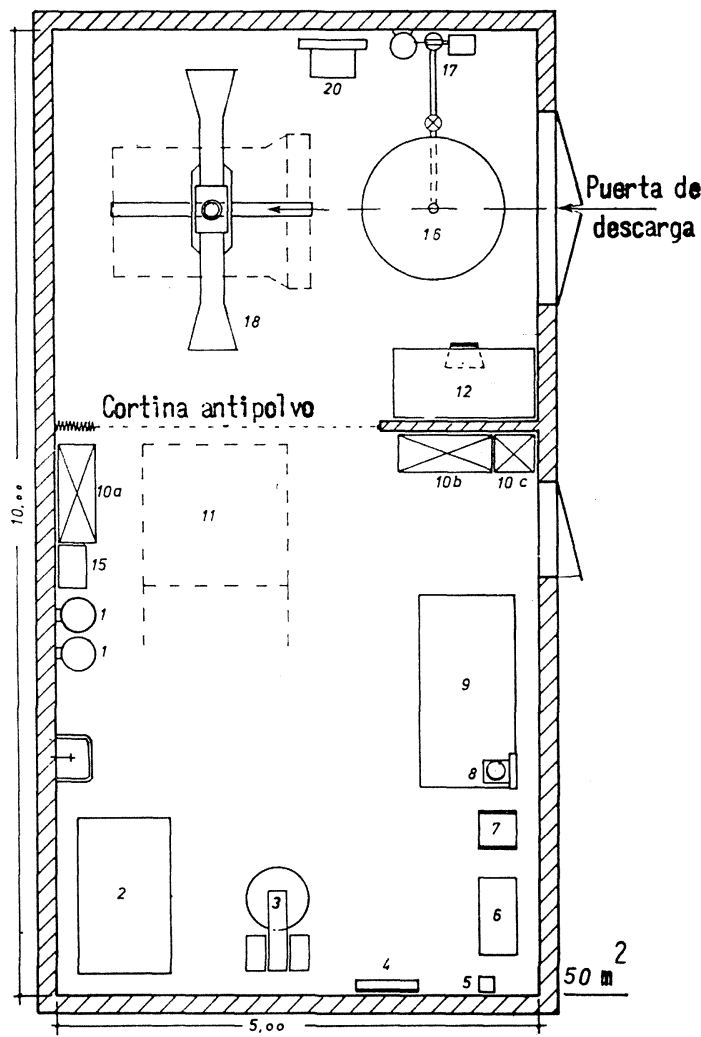

Fig. 4.-Laboratorio de ensayos para una fábrica de tubos. Tamaño del local: $50 \mathrm{~m}^{2}$.

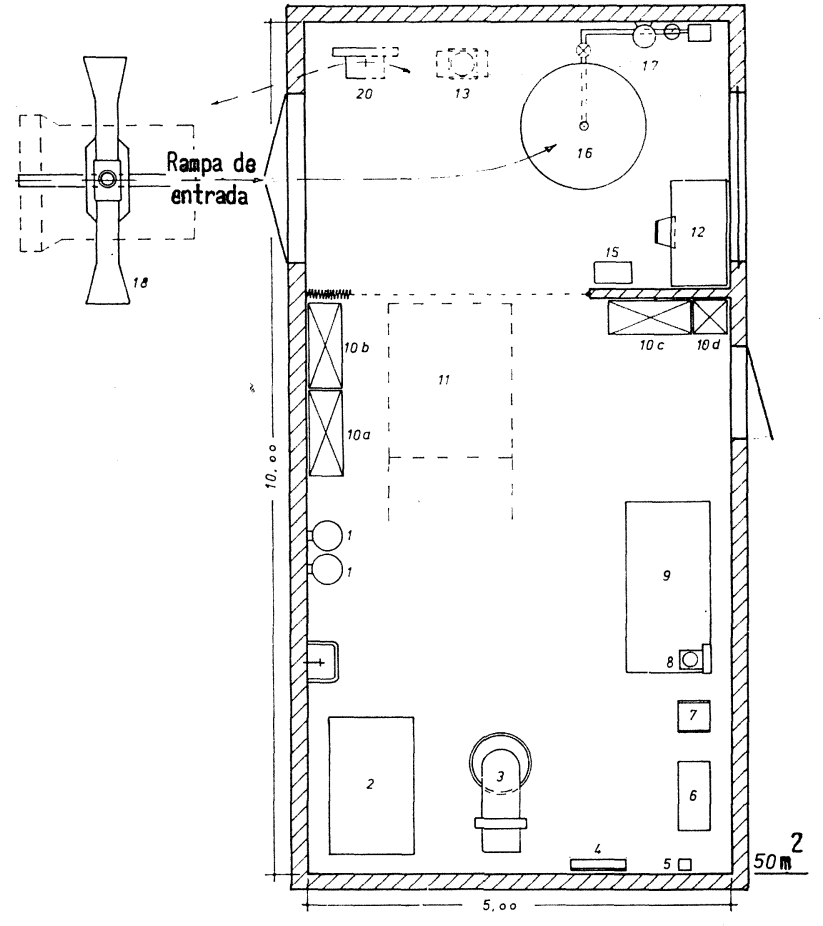

Fig. 5.-Otro laboratorio de ensayos para una fábrica de tubos. Tamaño del local: $50 \mathrm{~m}^{2}$.

El local de la fábrica semejante al anterior: Local sin polvo, con banco para pruebas de presión dentro del agua, 16/17. Máquina de ensayos de la presión de cresta, 18, fuera del local de comprobación. Máquina de ensayo de la presión, a elegir, 13, conforme DIN 4032 , proyecto diciembre 1971. Maniobra de las máquinas de ensayo mediante un pupitre de mando, 20.

Local de la fábrica análogo a los anteriores: El equipo de ensayo de los tubos, 18 y 16 , fuera del local bajo techo a dos vertientes. Máquina de ensayo de presión, 13, y en caso necesario, también máquina de ensayo de flexión, 14, en el local de pruebas. Pupitre de mando combinado, 20, para dos o tres máquinas.

Asistencia a varios ramos de producción o varias fábricas, por ejemplo: fábrica de bloques de hormigón, fábrica de tubos, fábrica de piezas prefabricadas, fábrica de hormigón preparado. 


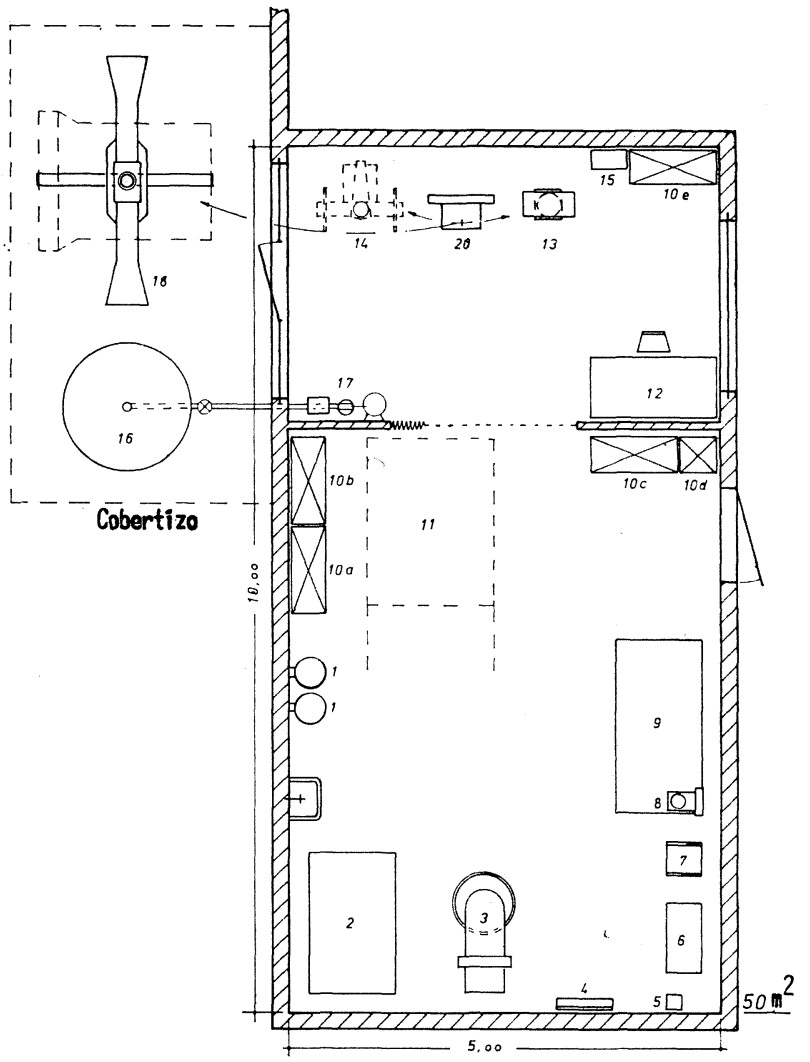

Fig. 6.-Laboratorio de ensayos para una fábrica de hormigón con producción mixta, incluidos tubos. Tamaño del local: $50 \mathrm{~m}^{2}$.

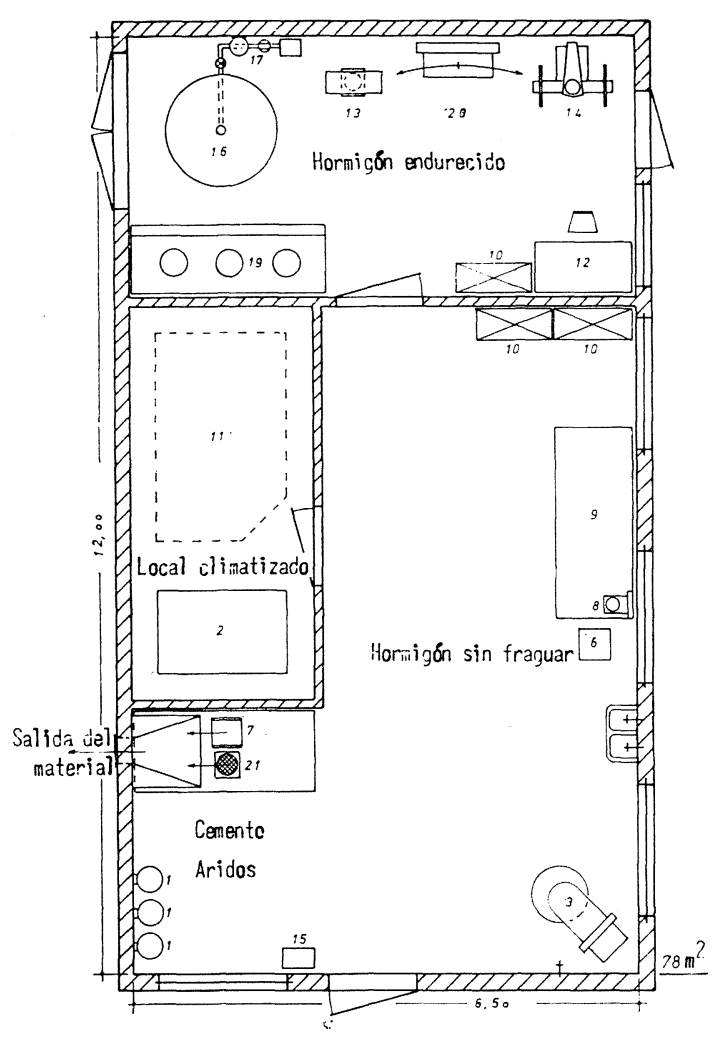

Fig. 7.-Laboratorio de ensayos de tamaño grande (también E): $78 \mathrm{~m}^{2}$.

Los trabajos con polvo en la zona inferior del gran local de la fábrica. Evacuación mecánica del polvo por medio de un tablero de trabajo adicional. Local climatizado separado por paredes con almacenamiento de aire y agua. Local sin polvo detrás de la pared divisoria, con los equipos necesarios para los ensayos del hormigón endurecido. Tres puertas exteriores facilitan los transportes de entrada y salida.

Asistencia a varios ramos de producción o fábricas (sin fábricas de tubos). Local suficientemente grande. Evacuación de polvo. Pequeña oficina con pared frontal acristalada para cálculos. Paredes divisorias fijas entre el local de la fábrica y el local climatizado por una parte, y local de ensayo por otra. Dos puertas exteriores.

En la fábrica el local con polvo está separado por una pared divisoria, flexible. Evacuación del polvo mediante un motor. Pequeña oficina situada en el centro con acristalamiento frontal y lateral para cálculos. Pared divisoria del local de ensayos. Pared divisoria del gran local climatizado para probetas de diferentes dimensiones y pesos. Ligero puente-grúa, 22, para la carga del recipiente de agua, del de almacenamiento y del vagón de transporte. Puertas exteriores propias para los transportes de acceso y salida. El proyecto de ampliación, indicado por rayas, prevé un local para muestras de reposición de cemento y un local de oficinas con uno o dos escritorios y ángulos de conversación (trato con los clientes). Posible desplazamiento del local de reposición al extremo del tramo de oficinas e instalación de servicios entre la oficina y el laboratorio. 
Todos los laboratorios de ensayo expuestos son ejemplos de ejecución, que pueden variar y modificarse de acuerdo con diferentes ideas.

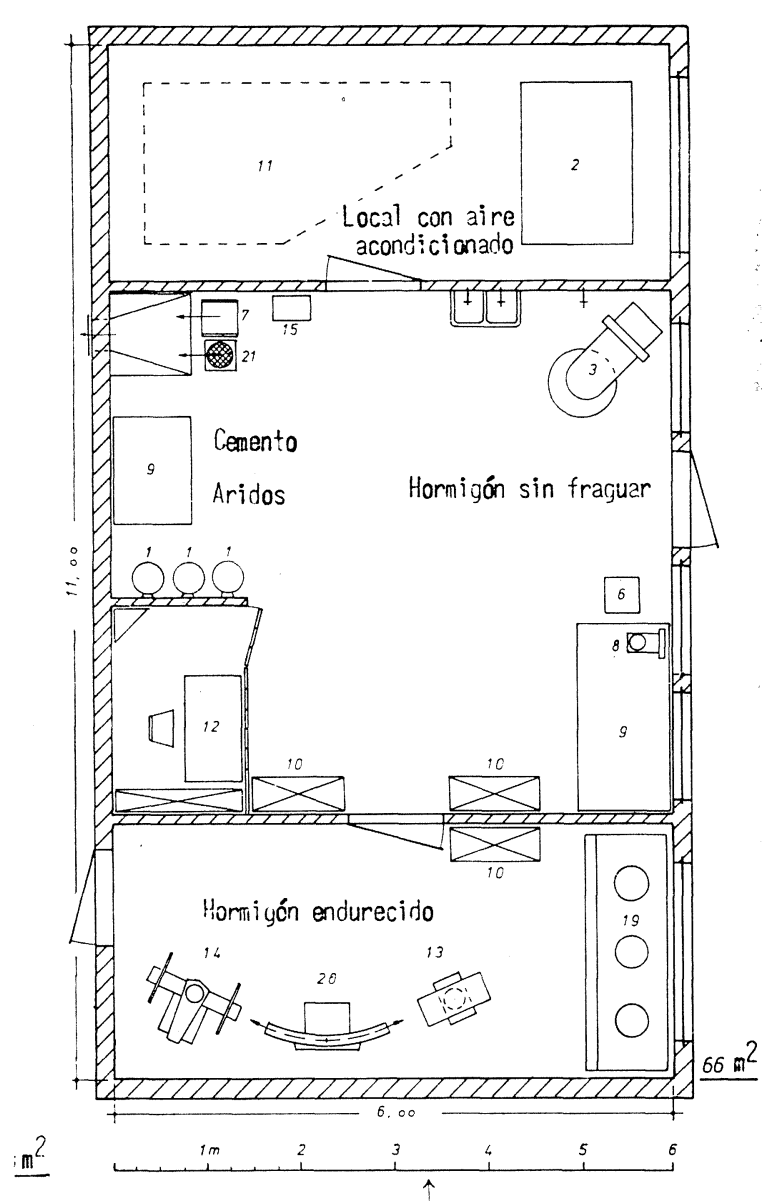

Fig. 8.-Otro laboratorio de ensayos de tamaño grande (también E): $66 \mathrm{~m}^{2}$.

Fig. 9.-Otro laboratorio más de ensayos de tamaño grande E: $91 \mathrm{~m}^{2}$.

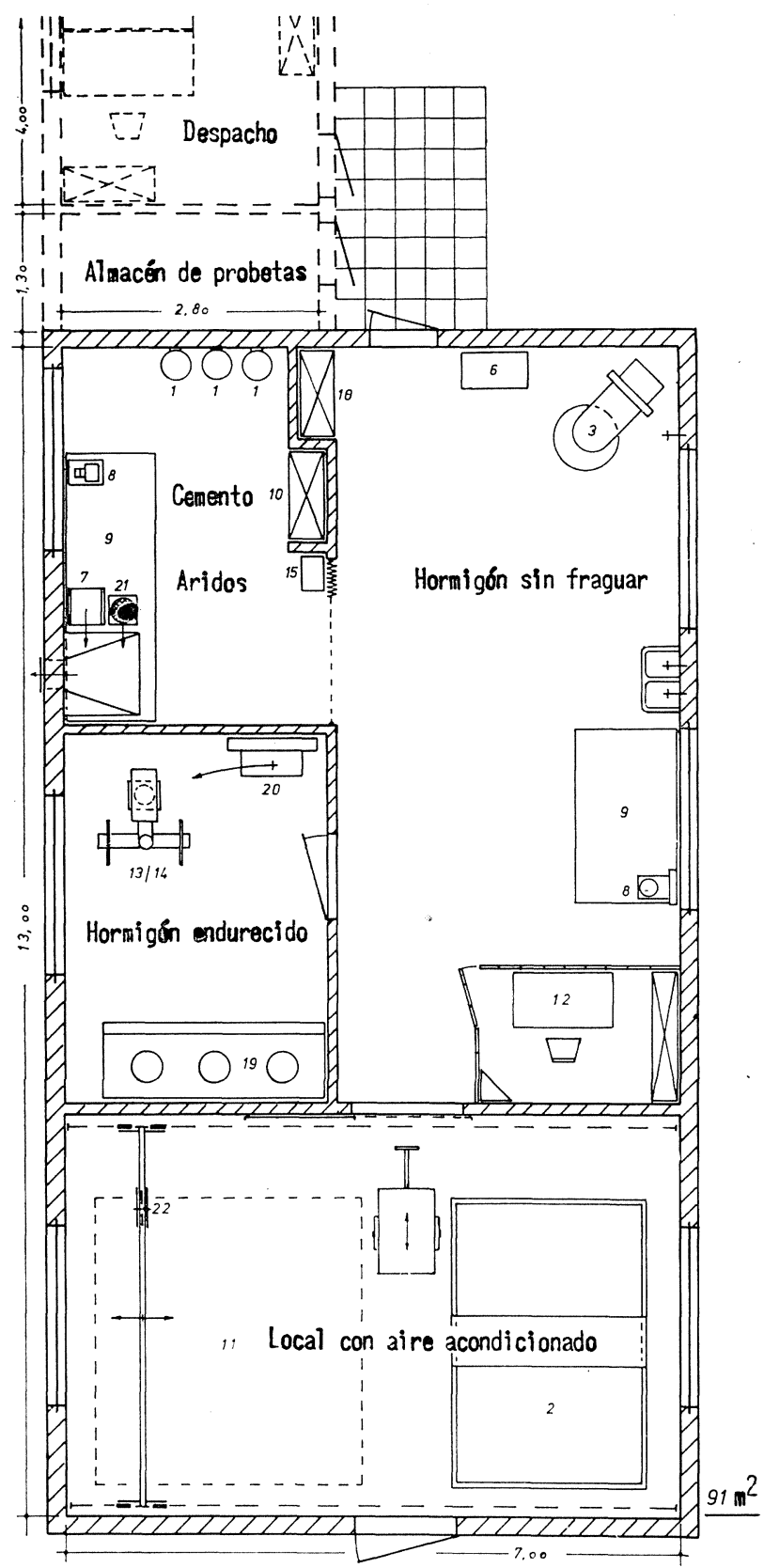

Quizás puedan ofrecer Vds. algunas sugerencias para la instalación de un laboratorio de ensayos propio o que lo lleven juntamente con otro interesado. Su tamaño debería regirse por la magnitud de los trabajos a realizar. El ensayador debe estar en situación de efectuar todos los trabajos de control y de ensayo existentes en un local suficientemente grande y bien equipado, situado en el centro de la fábrica. Incluso, contando con una economía reducida y una ampliación por etapas, debería tenerse en cuenta el tamaño defini- 
tivo, a ser posible desde el comienzo de la planificación. Las transformaciones o anexiones posteriores son costosas. No siempre constituyen un conjunto armónico con la antigua construcción.

\section{F) Reflexiones previas a la adquisición de equipo de ensayos}

Las máquinas y los aparatos de ensayos han de ser, con excepción de los equipos que dependen del desgaste, bienes económicos de larga duración. Los compradores no siempre ven en la relación correcta la calidad y el precio. Considerar cuidadosamente la adquisición de marcas baratas. Ejemplos:

\section{Latas de $5 \mathrm{~kg}$}

Para muestras de reposición de cemento se dan ya por un marco. Comprobar si estas latas son todavía estancas al aire después de frecuente abrir y cerrar las mismas. En caso de reclamación, la fábrica de cemento podría rechazar las muestras de cemento que se han aportado. Sólo se emplearán bolsas de plástico con el consentimiento del proveedor de cemento.

\section{Tamices}

Los tamices de modelo sencillo resisten años. Los tamices de gran calidad de material no oxidable y con fondo intercambiable se utilizan cuando hay que realizar muchos tamizados. El personal de laboratorio tiene sus propias ideas a este respecto.

No todos los materiales pasan, sin residuo, los tamaños inferiores. El personal cuidadoso de laboratorio tamiza posteriormente a mano. Para el tamizado previo se aplican como auxiliares, también, mesas vibradoras. Pero, a continuación, se debe girar el juego de tamices durante la vibración, porque los residuos del tamizado, especialmente tratándose de una gama de grano fino, se pegan a las paredes del tamiz de acuerdo con el sentido de giro del vibrador.

\section{Moldes}

Hay que revisar, en períodos regulares, la lisura de superficie de los moldes cúbicos. Todos se desgastan o deforman después de un uso prolongado. Aplicar la regla capilar. Mantener la placa contra la luz y girarla debajo de la regla capilar. Medir con el patrón la máxima ranura de luz. Las tolerancias mayores de $2 / 10 \mathrm{~mm}$ producen indicaciones defectuosas en más de $10 \%$. Volver a adquirir nuevos moldes o piezas moldeadas. Los moldes baratos con insuficiente lisura de superficie proporcionan cubos, cuyo poder informativo no permite comparación alguna con el hormigón incorporado. Deben cerrar en forma estanca los bastidores de asiento.

\section{Pesaje}

La balanza de platillos de la Asociación Alemana del Hormigón es exacta. Sin embargo, lleva mucho tiempo. El que quiere pesar mucho debe pensar, antes de la adquisición, si es más adecuada una balanza automática de gran calidad con panel indicador. El tiempo supone dinero. 
Aparatos de secado para áridos y hormigón no fraguado

Los equipos de propia construcción no funcionan siempre económicamente. Compruebe la oferta del mercado. Existen aparatos rentables con elevada capacidad de secado.

\section{Mezclador de laboratorio}

Tratándose de mezclas pequeñas me inclinaría por un mezclador forzado. No todo mezclador pequeño mezcla perfectamente una combinación de mezcla dada. La impresión óptica puede engañar. El mezclador de laboratorio debería producir la misma intensidad de mezcla que el gran mezclador de la fábrica.

\section{Mesas vibradoras}

Se ofrecen a diferentes precios. Examine el porqué. Un tablero de chapa con un vibrador atornillado y nervio distribuidor de vibración no es una mesa vibradora apta para un laboratorio. La construcción del tablero de la mesa debe permitir una radiación completamente homogénea de la vibración. Muchos prefieren mesas de vibración con frecuencia normal. Además de la radiación de la vibración, quieren aprovechar también el efecto de choque. Otros prefieren mesas con vibradores de alta frecuencia, tratándose especialmente de hormigón ligero. Los relojes avisadores aseguran, en la serie de ensayos, siempre iguales tiempos de vibración. Los moldes grandes y las combinaciones "secas" exigen tiempos de vibración más prolongados. Existen relojes avisadores para corriente alterna y corriente trifásica, con varios tiempos de ajuste: 0-6 ó 0-60 ó 0-360 segundos. En los análisis de series debería disponerse, por lo menos, de un cronómetro.

\section{Máquinas de ensayos}

Cada fabricante garantiza, actualmente, la contrastabilidad en la clase 3 ó 2 deseada. O bien se pueden adquirir calibrados o se pueden hacer calibrar inmediatamente después de su entrega. El fabricante es responsable. Sobre el dimensionamiento de la impulsión electrohidráulica no existe imposición alguna. Las bombas de alta presión y las válvulas de sobrepresión son muy sensibles. Su seguridad de servicio supone un filtrado perfecto del aceite. Las menores impurezas, por ejemplo por cascarilla de las tuberías de aceite, producen averías. Al proveedor que tiene máquinas de ensayos, al parecer muy ventajosas por su precio, debería hablársele de este problema. Los fábricantes de bombas de alta presión exigen filtros de aceite eficaces. $\mathrm{BOSCH}$, un sistema de bombas muy empleado, exige por ejemplo un filtro de fieltro, que sólo entrega aceite a la bomba de alta presión con utilización adicional de una bomba de transporte. Hoy en día debería ser evidente que las máquinas de ensayos de presión presentan ventajas de ensayos de fácil acceso, en las que no se aplastan los dedos.

\section{Resumen}

El autor, partiendo de la práctica, considera que el control propio es elemento del control de producción y del producto, que representa una instalación necesaria para asegurar la calidad de los productos y el rendimiento de la empresa. Valiéndose de ejemplos de control muestra posibilidades para mantener a un alto nivel la calidad de los productos, aumentar el rendimiento y reducir los costes con ayuda de equipos adecuados de ensayo. Diferentes distribuciones relativas al local de ensayos para sectores de trabajo pequeños y grandes ofrecen sugerencias para la instalación de una oficina propia de ensayos. 